\title{
LAS POSIBILIDADES PRESUPUESTARIAS DEL GOBIERNO EN FUNCIONES: A PROPÓSITO DE LA APROBACIÓN DE UN REAL DECRETO-LEY PARA LA MODIFICACIÓN DE LA LEY DE PRESUPUESTOS GENERALES DEL ESTADO ${ }^{1}$
}

\author{
Carmen Cámara Barroso y Eduardo Sanz Arcega \\ doi: http://dx.doi.org/10.18543/ed-65(1)-2017pp333-345
}

\begin{abstract}
SUMARIO: 1. INTRODUCCIÓN. 2. LÍMITES PARA LA APROBACIÓN DE UN REAL DECRETO-LEY QUe MODIFIQUe LA Ley De PRESUPUESTOS GeNeRALES DEL ESTADO. 3. DISCUSIÓN FINAL: UNA PROPUESTA INTERPRETATIVA PARA LA CONSTITUCIONALIDAD DE UNA MODIFICACIÓN DE LA LEY DE Presupuestos Generales del Estado a través de un REAl DeCReTO-LEY PROMULGADO POR UN GOBIERNO EN FUNCIONES.
\end{abstract}

\section{INTRODUCCIÓN}

La naturaleza provisional y teleológica que la Constitución española acoge del Gobierno en funciones (ex art. 101.2 CE) fundamenta una regulación de desarrollo que, «salvo casos de urgencia debidamente acreditados o por razones de interés general cuya acreditación expresa así lo justifique», restringe su capacidad de acción al despacho ordinario de asuntos -entre los que se entiende la facilitación del proceso de traspaso de poderes- (art. 21.3

${ }^{1}$ Los autores desean agradecer encarecidamente los comentarios de Sergio Luis Doncel Núñez, Profesor-tutor de la UNED, que han estimulado una mejora sustancial de la versión inicial. 
Ley 50/1997, de 27 de noviembre, del Gobierno)². Tan es así, que, en adición a la prohibición general expuesta $\mathrm{y}$, sin lugar a su excepción, la propia Ley del Gobierno proscribe la aprobación del Proyecto de Ley de Presupuestos Generales del Estado por parte de un Ejecutivo en funciones, así como la presentación por este de cualquier proyecto de ley ante las Cortes (ex art. 21.5 Ley del Gobierno).

No obstante lo anterior, la realidad puede dibujar escenarios que demanden una acción gubernativa sustancial, sobremanera por lo que hace al ámbito de la política económica. Ante una eventualidad semejante, las posibilidades presupuestarias de un Gobierno en funciones respecto de la modificación de los estados de ingresos y gastos -entre los que desde el punto de vista constitucional se incluye tanto la cuantificación de los beneficios fiscales que afecten a los tributos del Estado como el servicio de la deuda pública (respectivamente, arts. 134.2 y 135.3 CE)- consignados en la Ley de Presupuestos Generales del Estado (LPGE, en lo sucesivo) ${ }^{3}$ no quedarían limitadas respecto de las capacidades de un Gobierno con plenos poderes, sino en lo tocante a aquellas modificaciones que requieran de la presentación de un proyecto de ley ante las Cortes (de nuevo, ex art. 21.5 Ley del Gobierno).

La cuestión que, a renglón seguido, aflora, se refiere a la potestad que pudiere ostentar un Ejecutivo en funciones para aprobar una reforma de la LPGE a través de una norma con rango de ley que soslayase el requisito de presentación de un proyecto de ley ante las Cortes. Con otras palabras, la pregunta a la que, por el momento, no se le ha dado una solución legislativa concluyente, es la siguiente: ¿qué condiciones debe satisfacer un Gobierno en funciones para aprobar válidamente un real decreto-ley que modifique estados de gastos e ingresos contenidos en la LPGE que requieran para su modificación de la aprobación de una norma con rango de ley?

Para responder el interrogante planteado, y, con la motivación de extender la doctrina jurídica, el objetivo de este trabajo se orienta a la delimitación de las competencias del Gobierno en funciones, precisamente, en lo atinente

2 Más allá de la vinculación «al cumplimiento del principio y deber de lealtad constitucional» (Álvarez Conde 1996-1997, 195), la limitación de las capacidades del Gobierno en funciones ha sido fundamentada por la doctrina constitucional en «razones ligadas al propio concepto y naturaleza (figura transitoria, excepcional y de necesidad), posición (carencia, y falta de necesidad, de una legitimación parlamentaria, con legitimación directa excepcional ope constitutionis) y fin (el traspaso de poderes) de un Gobierno en funciones, y carente, por tanto, de todo respaldo parlamentario» (Brage Camazano y Reviriego 2006, 448).

${ }^{3}$ Pues el contenido de la Ley de Presupuestos debe limitarse a la ordenación jurídica del gasto público, contenido que, además, solo puede regularse a través de aquella (por todas; ilustrativamente, SSTC 3/2003, de 16 de enero, FJ 4, y 9/2013, de 28 de enero, FJ3). 
a la modificación de la LPGE a través de la promulgación de un real decretoley. Toda vez que la interposición de un recurso de inconstitucionalidad ante un real decreto-ley no suspende ni su vigencia ni su aplicación (ex art. 30 Ley Orgánica 2/1979, de 3 de octubre, del Tribunal Constitucional), y, dado que a un Gobierno en funciones no le asiste respaldo parlamentario alguno, parece oportuno ofrecer una interpretación reforzada para aceptar la constitucionalidad de las disposiciones con rango de ley que, en materia presupuestaria, pueda dictar un Gobierno en funciones. En lo que conocemos, si bien la realidad política española está motivando la proliferación de estudios acerca de las competencias del Gobierno en funciones, y que en relación con su capacidad para dictar un real decreto-ley subrayan como presupuestos habilitantes suficientes la circunscripción de su contenido dentro de los límites constitucionales y la efectiva urgencia de las circunstancias (ilustrativamente, García-Escudero 2016, 115; Menéndez Moreno 2017, 2), el análisis propuesto por este trabajo resulta una novedad doctrinal.

El artículo se estructura como sigue a continuación. En la siguiente sección se expone los requisitos que el ordenamiento y la jurisprudencia imponen como canon de constitucionalidad para la válida promulgación de un real decreto-ley, atendiendo singularmente a su capacidad para introducir modificaciones en la LPGE. La última sección concluye la necesidad de exigir requisitos adicionales a los que tradicionalmente viene demandando la jurisprudencia y la doctrina para que la promulgación por un Gobierno en funciones de un real decreto-ley que modifique la LPGE supere el test de constitucionalidad.

\section{LÍMITES PARA LA APROBACIÓN DE UN REAL DECRETO-LEY QUE MODIFIQUE LA LEY DE PRESUPUESTOS GENERALES DEL ESTADO}

La singularidad legislativa inherente a la aprobación de una norma con rango de ley por parte de un Ejecutivo se refleja en los requisitos formales y materiales que la Constitución impone para la validez jurídica de los reales decretos-leyes. De un lado, en relación con los requerimientos formales se exige la voluntad concurrente del Congreso de los Diputados para su convalidación o derogación «en el plazo de los treinta días siguientes a su promulgación» (arts. 86.2 y 86.3 CE, desarrollados por el art. 151 del Reglamento del Congreso de los Diputados de 10 de febrero de 1982, RCD en adelante). A fin de facilitar el trámite parlamentario, el propio Reglamento del Congreso señala expresamente que «la inserción en el orden del día de un Decreto-ley, para su debate y votación, podrá hacerse tan pronto como hubiere sido objeto de publicación en el Boletín Oficial del Estado» (art. 151.1 RCD).

En el caso de que el mandato del Congreso haya expirado o aquel se encuentre disuelto, «las Diputaciones Permanentes seguirán ejerciendo sus funciones 
hasta la constitución de las nuevas Cortes Generales» (art. 78.3 CE), si bien el Reglamento del Congreso estipula explícitamente que, en relación con los reales decretos-leyes, la competencia de la Diputación Permanente quedaría circunscrita a la posibilidad de «tramitar como proyectos de ley por el procedimiento de urgencia los Decretos-leyes que el Gobierno dicte durante los períodos entre legislaturas» (art. 151.5 RCD); posibilidad a la que también puede acogerse la Cámara durante el periodo de vigencia de su mandato, mas siempre y cuando el decreto-ley haya sido previamente convalidado (ex art. 151.4 RCD).

De otro lado, los requisitos materiales se refieren a la «extraordinaria y urgente necesidad» que debe presidir las circunstancias que motiven la aprobación de un real decreto-ley (ex art. 86.1 CE), así como a la interdicción de un buen número de materias que, en ningún caso, pueden ser objeto de regulación a través de aquel, a saber: ordenamiento de las instituciones básicas del Estado, derechos, deberes y libertades de los ciudadanos regulados en el Título I, régimen de las Comunidades Autónomas, y Derecho electoral general; reforma constitucional; y, por último, materias para cuya regulación se exija la aprobación de una ley orgánica (respectivamente, ex arts. 86.1, 167 y 168, y 81.2 CE). A continuación, se expone la doctrina del Tribunal Constitucional en relación con la apreciación del requisito de extraordinaria y urgente necesidad para, acto seguido, desarrollar los límites constitucionales que específicamente en materia financiera (tributario-presupuestaria), dado el objetivo de este trabajo, se impone al contenido de los reales decretos-leyes.

Por lo que a la concurrencia efectiva del presupuesto de una «extraordinaria y urgente necesidad» se refiere, «la jurisprudencia constitucional ha exigido desde sus inicios, [...], que la definición por los órganos políticos de una situación de «extraordinaria y urgente necesidad» sea explícita y razonada, y que exista una conexión de sentido o relación de adecuación entre la situación definida que constituye el presupuesto habilitante y las medidas que en el Decretoley se adoptan, de manera que estas últimas guarden una relación directa o de congruencia con la situación que se trata de afrontar» (STC 126/2016, de 7 de julio, FJ 5 $)^{4}$, considerando «adecuado el uso del Decreto-ley en situaciones que hemos denominado como «coyunturas económicas problemáticas», [...], para

${ }^{4}$ Con otras palabras, el Tribunal en el juicio de constitucionalidad analiza «dos elementos: a) en primer lugar, la presentación explícita y razonada de los motivos que han sido tenidos en cuenta por el Gobierno en su aprobación atendiendo a la exposición de motivos de la norma, al debate parlamentario de convalidación y, en su caso, al expediente de elaboración [«en este punto es un control externo, en el sentido de que debe verificar, pero no sustituir, el juicio político o de oportunidad que corresponde al Gobierno» (STC 182/1997, de 28 de octubre, FJ 3)]; b) en segundo lugar, la existencia de una necesaria conexión entre la situación de urgencia definida y la medida concreta adoptada para afrontarla» (STC 139/2016, de 12 de julio, FJ 4). 
cuyo tratamiento representa un instrumento constitucionalmente lícito, en tanto que pertinente y adecuado para la consecución del fin que justifica la legislación de urgencia» (STC 126/2016, de 7 de julio, FJ 3). Adicionalmente, el Tribunal ha manifestado que no es necesario que la «definición expresa de la extraordinaria y urgente necesidad haya de contenerse siempre en el propio real decreto-ley, sino que tal presupuesto cabe deducirlo igualmente de una pluralidad de elementos» (STC 139/2016, de 12 de julio, FJ 3).

A continuación, se describe los límites a la regulación mediante real decreto-ley de disposiciones de índole tributario-presupuestaria contenidas en la LPGE. En primer lugar, debe precisarse que la LPGE ordena el gasto público (ex art. 134.2 CE) -el cual comprende también el servicio de la deuda pública (ex art. 135.3 CE)-, si bien puede incorporar también, pero solo, aquellas medidas que «guarden una conexión económica o presupuestaria» (STC 9/2013, de 28 de enero, FJ 3) directa con aquel y que, además, «su inclusión esté justificada en el sentido de que sea un complemento necesario para la mayor inteligencia y para la mejor y más eficaz ejecución del Presupuesto y, en general, de la política económica del Gobierno» (STC 76/1992, de 14 de mayo, FJ 4). Dicho lo cual, la argumentación se centra específicamente, como se ha anunciado, en las disposiciones materialmente tributarias y presupuestarias contenidas en la LPGE.

En el ámbito tributario, la jurisprudencia constitucional parte de los límites enunciados en la cláusula general del artículo 86.1 CE, a partir de la cual explicita que «el Decreto-ley no puede alterar ni el régimen general ni los elementos esenciales del deber de contribuir. [...] Por tanto, no queda absolutamente impedida la utilización del Decreto-ley en materia tributaria, cuando concurre el supuesto habilitante como instrumento normativo del Gobierno al servicio de los objetivos de la política económica. Ahora bien, será preciso tener en cuenta en cada caso en qué tributo concreto incide el Decreto-ley -constatando su naturaleza, estructura y la función de capacidad económica-, qué elementos del mismo -esenciales o no, resultan alterados por este excepcional modo de producción normativa- $y$, en fin, cuál es la naturaleza y alcance de la concreta regulación de que se trate» (STC 182/1997, de 28 de octubre, FJ 7). En consecuencia, si se traslada la interpretación expuesta a la regulación de contenidos tributarios incluibles en la LGPE, y, teniendo en cuenta, además, que la Constitución señala expresamente que «la Ley de Presupuestos no puede crear tributos [;] podrá modificarlos cuando una ley tributaria sustantiva así lo prevea» (art. 134.7 CE), toda modificación mediante real decreto-ley de cláusulas tributarias insertas en la LPGE que se ajuste a los requerimientos formales y materiales antedichos superará el test de constitucionalidad, siempre y cuando se acepte que la norma con rango de ley que se requiere para modificar la LPGE no se agota en una ley formal (interpretación que parece consistente con la posición del 
Tribunal Constitucional ${ }^{5}$ ), pues «el real decreto-ley no se transforma en ley, es decir, no cambia su naturaleza jurídica» (STC 29/1982, de 31 de mayo, FJ 2), aunque el Congreso lo convalide.

Por otra parte, desde el punto de vista del gasto público, partiendo de que la Constitución española señala genéricamente que «el Gobierno podrá presentar proyectos de ley que impliquen aumento del gasto público o disminución de los ingresos correspondientes al mismo ejercicio presupuestario», siempre y cuando respete la prioridad de pago de los intereses y el capital de la deuda pública (respectivamente, ex arts. 134.5 y $135.3 \mathrm{CE})^{6}$, la jurisprudencia no ha establecido limitaciones concretas a la regulación mediante Decreto-ley de disposiciones atinentes a los estados de gastos, por lo que, mediando «extraordinaria y urgente necesidad», parece que se acepta la constitucionalidad de un real decreto-ley que contuviera modificaciones de índole presupuestaria (aceptando, de nuevo, que la modificación de la LPGE puede realizarse mediante una norma con rango de ley).

Así las cosas, la Constitución y la Ley 47/2003, de 26 de noviembre, General Presupuestaria (LGP, en lo sucesivo), como norma de desarrollo, relacionan un conjunto de requisitos formales y materiales para la válida modificación de los estados de gastos consignados en la LPGE. Requisitos que, fundamentalmente, se refieren tanto a la identificación del sujeto legitimado en última instancia para aprobar la modificación presupuestaria, como al rango de la norma jurídica a través de la cual pueda materializarse aquella. Por lo que hace al objetivo de este trabajo, el interés se centra en la identificación de modificaciones de la LPGE atinentes a los estados de gastos que precisen para su aprobación del consentimiento de las Cortes Generales ${ }^{7}$, modificaciones que, razonando la existencia de «extraordinaria y urgente necesidad», podrían efectuarse a través de un real decreto-ley.

De acuerdo con el ordenamiento jurídico, el Consejo de Ministros deberá remitir un proyecto de ley a las Cortes Generales en los casos siguientes:

a) «Cuando se trate de créditos extraordinarios y suplementos de crédito para atender obligaciones de ejercicios anteriores para las que no se

${ }^{5}$ La jurisprudencia constitucional ha resaltado el rango de ley de la LPGE, como ley en sentido pleno, aceptando la singularidad de su contenido material, como se ha puesto de relieve líneas atrás, pero sin impedir su modificación a través de un real decreto-ley (porque, como se expondrá a continuación, tampoco le ha sido requerido un pronunciamiento en tal sentido).

${ }^{6}$ De lo que se deduce directamente la imposibilidad de un Gobierno en funciones para acometer cambios por esta vía (de nuevo, ex art. 21.5 Ley del Gobierno).

${ }_{7}$ Acogiendo el principio general de que quid potest plus, potest minus, cualesquiera otras modificaciones únicamente requerirían el ejercicio de potestades del Ejecutivo y, en el caso del Gobierno en funciones, de la debida justificación (ex art. 21.3, Ley del Gobierno). 
anulara crédito en el ejercicio de procedencia, tanto si se financian con Fondo de Contingencia como con baja en otros créditos [este requisito también resulta de aplicación a esta tipología de créditos extraordinarios y suplementos de crédito del presupuesto de la Seguridad Social, ex artículo 57.5 LGP, y de los presupuestos de los organismos autónomos, ex artículo 56.7 LGP].

b) Cuando se trate de créditos extraordinarios o suplementarios para atender obligaciones del propio ejercicio cuando se financien con baja en otros créditos.

c) Cuando se trate de créditos extraordinarios o suplementarios que afecten a operaciones financieras del Presupuesto» (art. 55.2 LGP).

d) Cuando se trate de autorizar emisiones de deuda o contracciones de crédito (ex art. 135.3 CE), y cuyo reflejo en los presupuestos es inmediato, pues «los créditos para satisfacer los intereses y el capital de la deuda pública se entenderán siempre incluidos en el estado de gastos [del Presupuesto] (art. 135.3 CE); de ahí que «la LPGE [establezca] el límite de la variación del saldo vivo de Deuda del Estado de cada ejercicio presupuestario, al que se ajustarán las operaciones financieras que impliquen creación de Deuda» (art. 94 LGP). En adición a lo expuesto, $\mathrm{y}$, en términos generales, la habilitación normativa para emitir deuda suele contenerse en la propia LPGE.

e) Cuando se trate de enmendar o modificar los créditos para satisfacer los intereses y el capital de la deuda pública, [cuando estos] se ajusten a los dispuesto en la ley de emisión (ex art. 135.3 CE).

Sentado lo anterior, la cuestión que aflora, $\mathrm{y}$, a la que por el momento no se le ha dado una solución legislativa concluyente (ni jurisprudencial, por inexistencia de precedentes ${ }^{8}$ ), se refiere a si sería posible que un Gobierno en funciones modificase a través de un real decreto-ley disposiciones de la LPGE -tributarias o presupuestarias-que, de acuerdo con el literal de la regulación tributario-constitucional, precisarían de una norma con rango de ley para acometerse. Al efecto, y, en relación con un Gobierno con plenos poderes, el precedente que existe de una modificación de la LPGE a través de real decreto-ley (Real Decreto-Ley 11/2004, de 23 de diciembre, por el que se modifica, en materia de pensiones públicas, la Ley de Presupuestos Generales

${ }^{8}$ El primer precedente de un real decreto-ley promulgado por un Gobierno en funciones y recurrido ante el Tribunal Constitucional, que aún no se ha pronunciado, es el Real DecretoLey 1/2016, de 15 de abril, por el que se prorroga el Programa para la Activación del Empleo, al que se ha hecho referencia. Dicho real decreto-ley, que extiende los beneficios de aquel programa, contiene unos efectos presupuestarios inmediatos. Este decreto-ley fue convalidado por las Cortes (Resolución de 28 de abril de 2016, del Congreso de los Diputados). 
del Estado para 2005) fue convalidado por las $\operatorname{Cortes}^{9}$ y no recurrido ante el Tribunal Constitucional; teniendo en cuenta, además, que dicho decreto-ley se dictó aun antes de que la LPGE entrara en vigor, cuestión esta última que motivó la crítica de la doctrina (ilustrativamente, véase Falcón Tella, 2005).

De otro lado, el último precedente acerca de la promulgación de un real decreto-ley de contenido tributario por un Gobierno en funciones ${ }^{10}$ es el Real Decreto-Ley 2/2016, de 30 de septiembre, por el que se introducen medidas tributarias dirigidas a la reducción del déficit público, y que fue dictado arguyendo la concurrencia del requisito de extraordinaria y urgente necesidad derivada de la satisfacción del objetivo de déficit público fijado por las autoridades comunitarias que, al tiempo, avalaría tanto la disposición de un real decreto-ley como la satisfacción de idéntica condición para que un Gobierno en funciones pueda separarse de las limitaciones a su capacidad de acción ${ }^{11}$. De nuevo, el Tribunal Constitucional no ha tenido ocasión de pronunciarse sobre la constitucionalidad de dicho decreto-ley, que superó el proceso de convalidación por las $\operatorname{Cortes}^{12}$, lo que no fue obstáculo para que la doctrina criticase la dudosa presencia del presupuesto habilitante acerca de la concurrencia de extraordinaria y urgente necesidad ${ }^{13}$.

${ }^{9}$ Resolución de 28 de diciembre de 2004, del Congreso de los Diputados, por la que se ordena la publicación del Acuerdo de convalidación del Real Decreto-Ley 11/2004, de 23 de diciembre, por el que se modifica, en materia de pensiones públicas, la Ley de Presupuestos Generales del Estado para 2005.

${ }^{10}$ Como se ha puesto de relieve, el primer precedente de un real decreto-ley promulgado por un Gobierno en funciones es el Real Decreto-Ley 1/2016, de 15 de abril, por el que se prorroga el Programa para la Activación del Empleo.

${ }^{11}$ En la Exposición de Motivos se señala cómo «la inmediata reducción del déficit público justifica la adopción de las medidas que incorpora este real decreto-ley, concurriendo, de este modo, la circunstancia de extraordinaria y urgente necesidad que exige el artículo 86 de la Constitución Española para la utilización de dicha figura normativa, requisito imprescindible, como ha recordado, por otra parte, la jurisprudencia constitucional. La tramitación de este real decreto-ley se efectúa por un Gobierno en funciones, circunstancia que se ajusta a lo dispuesto por el artículo 21.3 de la Ley 50/1997, de 27 de noviembre, del Gobierno, precepto conforme al cual «el Gobierno en funciones facilitará el normal desarrollo del proceso de formación del nuevo Gobierno y el traspaso de poderes al mismo y limitará su gestión al despacho ordinario de los asuntos públicos, absteniéndose de adoptar, salvo casos de urgencia debidamente acreditados o por razones de interés general cuya acreditación expresa así lo justifique, cualesquiera otras medidas», siendo ese supuesto excepcional de urgencia el que concurre en esta ocasión» (Exposición de Motivos Real Decreto-Ley 2/2016).

${ }_{12}$ El Real Decreto-Ley expuesto fue convalidado mediante Resolución de 20 de octubre de 2016, del Congreso de los Diputados, por la que se ordena la publicación del Acuerdo de convalidación del Real Decreto-Ley 2/2016, de 30 de septiembre, por el que se introducen medidas tributarias dirigidas a la reducción del déficit público.

${ }_{13}$ In extenso, se ha criticado que «el Real Decreto-Ley 2/2016, además de tener alcance retroactivo, incorpora a nuestro ordenamiento una modificación intertemporal, esto es, 
Llegados a este punto, a nuestro juicio, la superación del test de constitucionalidad de los reales decretos-leyes que modifiquen disposiciones atinentes a la LPGE debería satisfacer requisitos adicionales a los relatados en esta sección, referidos estos, tal y como se ha expuesto, tanto a la ambición del contenido que se regule como a la apreciación de la urgencia y extraordinariedad de las circunstancias que motiven su aprobación.

\section{DISCUSIÓN FINAL: UNA PROPUESTA INTERPRETATIVA PARA LA CONSTITUCIONALIDAD DE UNA MODIFICACIÓN DE LA LEY DE PRESUPUESTOS GENERALES DEL ESTADO A TRAVÉS DE UN REAL DECRETO-LEY PROMULGADO POR UN GOBIERNO EN FUNCIONES}

El ciclo económico-electoral puede dibujar escenarios que demanden de un Gobierno en funciones la toma de decisiones sustantivas, sobremanera por lo que hace al ámbito de la política presupuestaria. Toda vez que respecto de las capacidades de un Gobierno en funciones, mediando una eventualidad subsumible en el supuesto de «casos de urgencia debidamente acreditados o por razones de interés general cuya acreditación expresa así lo justifique» (art. 21.3 Ley del Gobierno), es un lugar común afirmar que aquellas quedarían limitadas única y formalmente a la presentación de proyectos de ley ante las Cortes, así como a la aprobación del Proyecto de Ley de Presupuestos Generales del Estado (ex art. 21.5 Ley del Gobierno). La cuestión que aflora, objetivo de este trabajo, y que, en lo que conocemos, no ha sido abordada, es la siguiente: ¿qué condiciones debe satisfacer un Gobierno en funciones para aprobar válidamente un real decreto-ley que modifique estados de gastos e ingresos contenidos en la LGPE que requieran para su modificación de la aprobación de una norma con rango de ley?

$\mathrm{Al}$ efecto, en la sección anterior se ha identificado los requisitos que el ordenamiento y la jurisprudencia imponen para la válida promulgación de un real decreto-ley en materia financiera, así como las modificaciones de la LPGE que demandan para ser efectivas de una norma con rango de ley. Específicamente por lo que hace a los primeros, mediando «extraordinaria y urgente necesidad», la jurisprudencia avala la regulación mediante real decreto-ley de normas tributarias y presupuestarias, siempre, respecto de las primeras, que no se desnaturalice la reserva de ley tributaria prevista constitucionalmente (ex art. 86.1 CE).

sistémica, no coyuntural ni sujeta, por tanto, a alguna condición específica de esa coyuntura que limite su vigencia, y por lo tanto difícilmente reconducible solo a las necesidades que provocan la extraordinaria y urgente necesidad que requiere la forzosamente coyuntural regulación mediante Decreto-ley, cuando este no se tramita después como ley ordinaria» (Menéndez Moreno 2017, 5). 
Llegados a este punto, la singularidad asociada a la aprobación por el Gobierno de una norma con rango de ley soslaya, al menos temporalmente, la función legislativa encomendada constitucionalmente a las Cortes Generales, las cuales, en relación con los Presupuestos Generales del Estado, «cumplen tres objetivos especialmente relevantes: a) aseguran, en primer lugar, el control democrático del conjunto de la actividad financiera pública (arts. 9.1 y 66.2, ambos de la Constitución); b) participan, en segundo lugar, de la actividad de dirección política al aprobar o rechazar el programa político, económico y social que ha propuesto el Gobierno y que los presupuestos representan; c) controlan, en tercer lugar, que la asignación de los recursos públicos se efectúe, como exige expresamente el art. 31.2 CE, de una forma equitativa, pues el presupuesto es, a la vez, requisito esencial y límite para el funcionamiento de la Administración» (STC 3/2003, de 16 de enero, FJ 4). De ahí que, a nuestro juicio, la exigencia de requisitos adicionales no se encamina sino, precisamente, al refuerzo de la función constitucional que, en relación con la aprobación de los Presupuestos, ostentan las Cortes Generales.

A tal fin, y, en primer lugar, debe diferenciarse cuatro escenarios ante los que un Gobierno en funciones podría, de acuerdo con la interpretación de las circunstancias en las que se desenvuelve la realidad económica, promulgar un real decreto-ley para modificar contenidos de la LPGE que requieren para su validez de la aprobación de una norma con rango de ley: a) Cortes disueltas y presupuestos aprobados; b) Cortes disueltas y presupuestos prorrogados; c) Cortes constituidas y presupuestos aprobados; d) Cortes constituidas y presupuestos prorrogados. Mientras la distinción entre la existencia de Cortes disueltas o constituidas se motiva por la centralidad de la función legislativa en relación con la aprobación de los presupuestos; la normativa sobre estabilidad presupuestaria abre a nuestro juicio la posibilidad de que un Ejecutivo en funciones pueda interpretar la incursión en un escenario de prórroga presupuestaria como situación de extraordinaria y urgente necesidad, esto es, como presupuesto habilitante válido para la promulgación de un real decreto-ley. Así, un escenario de prórroga presupuestaria se caracteriza porque «la prórroga no afectará a los créditos para gastos correspondientes a programas o actuaciones que terminen en el ejercicio cuyos presupuestos se prorrogan o para obligaciones que se extingan en el mismo» (art. 38.2 LGP). Si se conjuga lo anterior con la regla de gasto que impide el crecimiento del gasto público por encima del crecimiento del Producto Interior Bruto nominal, previsión contenida en la Ley Orgánica 2/2012, de 27 de abril, de Estabilidad Presupuestaria y Sostenibilidad Financiera (ex art. 12.1), los efectos de una prórroga presupuestaria pueden precipitar una redimensión a la baja y permanente del tamaño del Sector Público a futuro, lo que, a nuestro juicio, parece presupuesto habilitante suficiente, en el sentido del artículo 86.1 CE, para la promulgación de un real decreto-ley. 
De la misma manera, respecto del supuesto de autorizar nuevas emisiones de deuda, dado que, en términos generales, dichas autorizaciones se realizan en el seno de la LPGE, en el supuesto de que el Fondo de Contingencia hubiera agotado sus recursos y/o las medidas requeridas superasen los supuestos de empleo de recursos con cargo a aquel ${ }^{14}$, entendemos que este hecho también constituiría un supuesto habilitante suficiente para que, mediante real decreto-ley, se pudiese autorizar tal emisión de deuda.

Sentado lo anterior, a continuación, se detalla los requisitos adicionales que debería exigirse en cada uno de los cuatro escenarios detallados para que un eventual real decreto-ley superase el test de constitucionalidad. En el caso de que las Cortes se encuentren disueltas y los presupuestos del ejercicio estuvieran aprobados, el riesgo de prórroga debe aceptarse como presupuesto habilitante para realizar una modificación en la LPGE, que podrá hacerse efectiva acudiendo a la figura del real decreto-ley, si se trata de realizar modificaciones que requieren de una norma con rango de ley (entre las que se incluye la autorización para emitir deuda pública o contraer crédito). En este sentido, el carácter de ultima ratio exigible respecto de las modificaciones de la LPGE que requieren de una norma con rango de ley aconseja, para que efectivamente así sea, imponer un límite cuantitativo a las modificaciones señaladas, ilustrativamente el importe del Fondo de Contingencia.

Con todo, a nuestro juicio la potencial incursión en una situación de prórroga avalaría la utilización de la figura del real decreto-ley para incrementar el Fondo de Contingencia en la cuantía de los créditos del Presupuesto que, en caso de prórroga, no puedan ser objeto de aquella. No en vano, a tenor del principio de reserva de ley presupuestaria, entendemos que no debería aceptarse que esta última medida fuera llevada a cabo a través de las atribuciones reglamentarias del Ejecutivo.

Por último, $\mathrm{y}$, en todos los casos descritos, la aprobación de un real decreto-ley deberá realizarse menos de treinta días antes de que las nuevas Cortes se constituyan, a fin de no soslayar la preceptiva fiscalización parlamentaria. A este respecto también contribuiría, de lege ferenda, una disminución del plazo de constitución de las nuevas Cortes tras las elecciones.

${ }^{14}$ De acuerdo con la normativa presupuestaria, el Fondo únicamente financiará «las ampliaciones de crédito reguladas en el artículo 54, los créditos extraordinarios y suplementos de crédito, de conformidad con lo previsto en el artículo 55, y las incorporaciones de crédito, conforme al artículo 58, [a salvo de que se trate de] modificaciones relativas al pago de la Deuda Pública, créditos destinados a financiar a las Comunidades Autónomas y Entidades Locales en aplicación de sus respectivos sistemas de financiación, así como a las que no reduzcan la capacidad de financiación del Estado en el ejercicio, computadas en la forma establecida en el artículo 27 de la Ley Orgánica 2/2012, de 27 de abril, de Estabilidad Presupuestaria y Sostenibilidad Financiera» (respectivamente, arts. 50 y 59 LGP). 
En segundo lugar, si las Cortes se hallan disueltas y los presupuestos están prorrogados, operarían dos de los límites planteados en el escenario anterior. De un lado, el real decreto-ley deberá ser promulgado durante los treinta días anteriores a la constitución de las nueva Cortes. De otro lado, el límite cuantitativo de las modificaciones no deberá superar el valor monetario del Fondo de Contingencia.

En tercer lugar, en el caso de que las Cortes se encuentren constituidas y los presupuestos estén aprobados, el real decreto-ley debería convalidarse al día siguiente de su publicación en el Boletín Oficial del Estado. De otro lado, y, de nuevo, el riesgo de incursión en una situación de prórroga presupuestaria permitiría alterar la LPGE en la cuantía de los créditos que no puedan ser objeto de prórroga, siempre y cuando su importe se destine a engrosar el Fondo de Contingencia; así como también los aspectos de la LGPE que requieran de una norma con rango de ley para ser modificados (con el límite del antedicho Fondo), entre los que se cuenta la autorización para emitir deuda pública.

Por último, si las Cortes están constituidas y los presupuestos se encuentran prorrogados, la validez de un hipotético real decreto-ley requiere que su convalidación se realice al día siguiente de su publicación en el Boletín Oficial del Estado, al tiempo que el límite cuantitativo de las modificaciones presupuestarias introducidas en su articulado no deberá superar el importe del Fondo de Contingencia.

Así las cosas, y, a modo de corolario, la naturaleza provisional del Gobierno en funciones, del mismo modo que su carencia de respaldo parlamentario, avalan la regulación restrictiva que el ordenamiento realiza de sus competencias. La realidad económica, empero, puede personificar escenarios ante los que sea urgente la toma de decisiones sustantivas en el ámbito de la política presupuestaria. Entendiendo que la inacción en contextos de extraordinaria y urgente necesidad puede precipitar resultados significativamente más perniciosos que la toma de una decisión gubernativa sustantiva, cual resulta la promulgación de un real decreto-ley, este trabajo defiende la capacidad de un Gobierno en funciones para modificar la LPGE a través de un real decreto-ley, siempre y cuando se satisfagan los requerimientos adicionales desarrollados líneas arriba y que, en lo que conocemos, constituyen una extensión de la doctrina y de la jurisprudencia constitucionales ${ }^{15}$. No en vano, la promulgación de un real decreto-ley excede del cometido natural de un Gobierno en funciones que, en todo caso, debe quedar presidido por la lealtad constitucional hacia el nuevo Ejecutivo que nace de la voluntad renovada de la ciudadanía expresada en las elecciones.

${ }^{15} \mathrm{Si}$ bien el razonamiento expuesto resulta sustancialmente extrapolable a los Gobiernos autonómicos en funciones, el análisis del escenario regional escapa a la ambición de este trabajo, por lo que resulta una oportunidad para la realización de una futura investigación. 
TITLE: The budgetary possibilities of the temporary government: on propose of the approval of a royal decree-law for the amendment of the National Budget Act

RESUMEN: El objetivo de este trabajo es extender la doctrina jurídica sobre las competencias presupuestarias del Gobierno en funciones, concretamente, en lo atinente a su capacidad para modificar los Presupuestos Generales del Estado a través de la promulgación de un real decreto-ley. A tal fin, en primer lugar, se expone los requisitos que el ordenamiento y la jurisprudencia imponen como canon de constitucionalidad para la válida promulgación de un real decreto-ley, atendiendo singularmente su capacidad para introducir modificaciones en la Ley de Presupuestos Generales del Estado (LGPE). La última sección concluye la necesidad de exigir requisitos adicionales para que la promulgación por un Gobierno en funciones de un real decreto-ley que modifique la LPGE supere el test de constitucionalidad.

The budgetting possibilities of the temporary government: on propose of the approbation of a real decree-law for the modification of the General Budget Law of the State.

Palabras Clave: Gobierno en funciones, Ley de Presupuestos Generales del Estado, real decreto-ley, Test de constitucionalidad, Cortes Generales.

ABSTRACT: The objective of this work is to extend the legal doctrine about the budgeting competences of the temporary government, especially, in which concerns to the capability to modify the General Budgets of the State through the promulgation of a decree-law. With this goal, in first place, the requisites that the set of laws and the jurisprudence impose are presented as cannon of constitutionality for the valid promulgation of Real Decree-Law, attending in singularity for their capacity to introduce modifications in the National Budget Act. The last section concludes the necessity of demand additional requisites for the promulgation for a temporary government of a Royal decree-law that modifies the National Budget Act.

KEY WORDS: Temporary Government, National Budget Act, Royal Decree-Law, Constitutionality Test, Spanish Parliament. 